\title{
INEQUALITIES FOR HOLOMORPHIC FUNCTIONS OF SEVERAL COMPLEX VARIABLES
}

BY

\author{
JACOB BURBEA ${ }^{1}$
}

\begin{abstract}
Sharp norm-inequalities, valid for functional Hilbert spaces of holomorphic functions on the polydisk, unit ball and $\mathbf{C}^{n}$ are established by using the notion of reproducing kernels. These inequalities extend earlier results of Saitoh and ours.
\end{abstract}

1. Introduction. In this paper we establish sharp norm-inequalities, valid for functional Hilbert spaces of holomorphic functions on the polydisk, unit ball and $\mathrm{C}^{n}$, by using the theory of reproducing kernels. Several applications, notably in the Dirichlet, Bergman-Selberg and Fischer spaces, are given. These inequalities constitute an improvement and an extension of recent results of Saitoh $[7,8]$, which he obtained by using methods different from ours. They also extend our earlier results in $[2,3]$ for the one complex variable case. The proofs given here employ elementary means and the results obtained may also be applied to various other situations where the domain in question is one of the classical Cartan domains. However, in order to avoid lengthy discussions, we shall not pursue the extensions here.

2. Preliminaries and notation. We consider the $n$-dimensional complex space $\mathbf{C}^{n}$ with the usual inner product

$$
\langle z, \zeta\rangle=\sum_{j=1}^{n} z_{j} \bar{\zeta}_{j} \quad\left(z, \zeta \in \mathbf{C}^{n}\right)
$$

and the associated norm $|z|=\langle z, z\rangle^{1 / 2}$. The unit ball $B \equiv B_{n}$ of $\mathbf{C}^{n}$ is then the set of all $z \in \mathbf{C}^{n}$ with $|z|<1$. The boundary of $B$ is the sphere $S \equiv S_{n}$, the set of all $z \in \mathbf{C}^{n}$ with $|z|=1$. By $P$ we denote the polydisk $B_{1}^{n}$. Sometimes it is convenient to use the notation of $D_{\rho}, \rho=0,1, \infty$, to refer to $P$ when $\rho=0$, to $B$ when $\rho=1$ and to $\mathbf{C}^{n}$ when $\rho=\infty$. The class of all holomorphic functions in $D_{\rho}$ is denoted by $H\left(D_{\rho}\right)$. It is a linear space over $\mathbf{C}$. The following standard multi-index notation is employed: If $\alpha=\left(\alpha_{1}, \ldots, \alpha_{n}\right) \in \mathbf{Z}_{+}^{n}$ (i.e., an $n$-tuple of nonnegative integers), then $|\alpha|=\alpha_{1}+\cdots+\alpha_{n}$ and $\alpha !=\alpha_{1} ! \cdots \alpha_{n} !$. In addition, for $z \in \mathbf{C}^{n}$, the notation $z^{\alpha}$ is an abbreviation for the holomorphic monomial $z_{1}^{\alpha_{1}} \cdots z_{n}^{\alpha_{n}}$. By $e_{k} \in \mathbf{Z}_{+}^{n}(1 \leqslant k \leqslant n)$ we mean the $n$-tuple that has 1 in the $k$ th entry and 0 everywhere else.

Any $f \in H\left(D_{\rho}\right)$ can be represented as

$$
f(z)=\sum_{\alpha} a_{\alpha} z^{\alpha} ; \quad a_{\alpha} \in \mathbf{C}, z \in D_{\rho},
$$

Received by the editors May 18, 1981 and, in revised form, February 22, 1982.

1980 Mathematics Subject Classification. Primary 32H10, 46E20.

${ }^{1}$ The author wishes to acknowledge valuable comments by the referee. 
where the series expansion converges absolutely and uniformly on compacta of $D_{\rho}$. In this way we regard $H\left(D_{\rho}\right)$ as the span of all $z^{\alpha}, \alpha \geqslant 0$, in the uniform topology of $D_{\rho}$.

We introduce a "derivation-operator" $\partial_{n}: H\left(D_{\rho}\right) \rightarrow H\left(D_{\rho}\right)$ by

$$
\partial_{n} f(z)=\sum_{\alpha \geqslant 1} \pi(\alpha) a_{\alpha} z^{\alpha-1} .
$$

The quantities in this sum are defined as follows: For $\alpha \geqslant 1$, let $\alpha_{k_{1}}, \ldots, \alpha_{k_{m}}$, $1 \leqslant k_{1} \leqslant \cdots \leqslant k_{m} \leqslant n(1 \leqslant m \leqslant n)$ be the nonzero $(\geqslant 1)$ components of $\alpha$. Then

$$
z^{\alpha-1}=z_{k_{1}}^{\alpha_{k_{1}}-1} \cdots z_{k_{m}}^{\alpha_{k_{m}}-1}
$$

and

$$
\pi(\alpha)=\left\{\left(\prod_{j=1}^{m} \alpha_{k_{j}}\right) \frac{(|\alpha|+n-m) !}{(|\alpha|-1) !}\right\}^{1 / 2}, \quad \alpha \geqslant 1 .
$$

This derivation $\partial_{n}$ is a linear operator of $H\left(D_{\rho}\right)$ into itself and constitutes the $n$-dimensional generalization of the usual derivative $\partial \equiv \partial_{1}$ on $H\left(D_{\rho}\right)$ when $n=1$. In fact, when $n=1, \partial_{1} f=f^{\prime}$ for $f \in H\left(D_{\rho}\right)$.

By $d \sigma(z)$ we denote the Lebesgue measure on $\mathbf{C}^{n}=\mathbf{R}^{2 n}$, and by $d s(z)$ we denote the euclidean measure on the boundary $S$ of $B$. For future reference we also record the following elementary identities (see, for example, [6, pp. 16-17, 120-121]): For $q>0$ and $\alpha, \beta \in \mathbf{Z}_{+}^{n}$ we have

$$
\frac{1}{\pi^{n}} \int_{B} z^{\alpha} \bar{z}^{\beta}\left(1-|z|^{2}\right)^{q-1} d \sigma(z)=\frac{\alpha ! \Gamma(q)}{\Gamma(q+|\alpha|+n)} \delta_{\alpha \beta}
$$

and

$$
\frac{1}{2 \pi^{n}} \int_{S} z^{\alpha} \bar{z}^{\beta} d s(z)=\frac{\alpha !}{\Gamma(|\alpha|+n)} \delta_{\alpha \beta} .
$$

3. Dirichlet and Bergman-Selberg spaces. For a fixed $q>0$ we write

$$
q(n) \equiv(n+1) q / 2 \text {. }
$$

The " $(n, q)$-Dirichlet space" is defined by

$$
\mathscr{D}_{n, q}=\left\{f \in H(B): f(0)=0,\|f\|_{\mathscr{D}_{n, q}}<\infty\right\},
$$

where

$$
\|f\|_{\mathscr{D}_{n, q}}^{2}=\frac{1}{\pi^{n} q(n)} \int_{B}\left|\partial_{n} f(z)\right|^{2} d \sigma(z) .
$$

Let $f \in \mathscr{\mathscr { D }}_{n, q}$ so that

$$
f(z)=\sum_{\alpha \geqslant 1} a_{\alpha} z^{\alpha}, \quad z \in B
$$

By (2.1)-(2.4) and (3.1),

$$
\begin{aligned}
\|f\|_{\mathscr{D}_{n, q}}^{2} & =\frac{1}{\pi^{n} q(n)} \sum_{\alpha \geqslant 1}|\pi(\alpha)|^{2}\left|a_{\alpha}\right|^{2} \int_{B} z^{\alpha-1} \bar{z}^{\alpha-1} d \sigma(z) \\
& =\frac{1}{q(n)} \sum_{\alpha \geqslant 1}|\pi(\alpha)|^{2}\left|a_{\alpha}\right|^{2} \frac{(\alpha-1) !}{(|\alpha|+n-m) !},
\end{aligned}
$$


where $\alpha_{k_{1}}, \ldots, \alpha_{k_{m}}, 1 \leqslant k_{1} \leqslant \cdots \leqslant k_{m} \leqslant n(1 \leqslant m \leqslant n)$ are the nonzero components of $\alpha \geqslant 1$. It follows that

$$
\|f\|_{\Phi_{n, q}}^{2}=\frac{1}{q(n)} \sum_{\alpha \geq 1} \frac{\alpha !}{(|\alpha|-1) !}\left|a_{\alpha}\right|^{2} .
$$

Moreover, by the Cauchy-Schwarz inequality

$$
|f(z)|^{2}=\left|\sum_{\alpha \geqslant 1} a_{\alpha} z^{\alpha}\right|^{2} \leqslant\left[q(n) \sum_{\alpha \geqslant 1} \frac{(|\alpha|-1) !}{\alpha !} z^{\alpha} \bar{z}^{\alpha}\right]\|f\|_{\mathscr{D}_{n, q}}^{2} .
$$

The quantity in the square bracket is $-q(n) \log \left(1-|z|^{2}\right)$, as the computation below shows, and therefore

$$
|f(z)| \leqslant\left\{-q(n) \log \left(1-|z|^{2}\right)\right\}^{1 / 2}\|f\|_{\mathscr{D}_{n, q}}, \quad z \in B .
$$

This shows that $\mathscr{D}_{n, q}$ is a functional Hilbert space of holomorphic functions $f$ in $B$ with $f(0)=0$. The space $\mathscr{D}_{n, q}$ has a reproducing kernel $k_{n, q}(\cdot, \cdot)$ which may be computed by means of any orthonormal basis of $\mathscr{D}_{n, q}$. Clearly the sequence

$$
\phi_{\alpha}(z)=\{q(n)(|\alpha|-1) ! / \alpha !\}^{1 / 2} z^{\alpha}, \quad \alpha \geqslant 1,
$$

is orthonormal in $\mathscr{W}_{n, q}$, and by (3.2) it is also complete. Therefore,

$$
\begin{aligned}
k_{n, q}(z, \zeta) & =\sum_{\alpha \geqslant 1} \phi_{\alpha}(z) \overline{\phi_{\alpha}(\zeta)}=q(n) \sum_{\alpha \geqslant 1} \frac{(|\alpha|-1) !}{\alpha !} z^{\alpha \bar{\zeta}} \\
& =q(n) \sum_{\alpha \geqslant 1} \frac{1}{\alpha !}\left(\sum_{m=|\alpha|} \frac{1}{m} m !\right) z^{\alpha \bar{\zeta} \alpha}=q(n) \sum_{m=1}^{\infty} \frac{1}{m}\left(\sum_{|\alpha|=m} \frac{m !}{\alpha !} z^{\alpha \bar{\zeta}^{\alpha}}\right) \\
& =q(n) \sum_{m=1}^{\infty} \frac{1}{m}\langle z, \zeta\rangle^{m},
\end{aligned}
$$

whence

$$
k_{n, q}(z, \zeta)=-q(n) \log (1-\langle z, \zeta\rangle) ; \quad z, \zeta \in B .
$$

Another related functional Hilbert space is the so-called " $(n, q)$-Bergman-Selberg space," which is defined by

$$
\mathcal{H}_{n, q}=\left\{g \in H(B):\|g\|_{n, q}<\infty\right\},
$$

where, for $g \in H(B)$ with

$$
\begin{gathered}
g(z)=\sum_{\alpha \geqslant 0} b_{\alpha} z^{\alpha}, \quad z \in B, \\
\|g\|_{n, q}^{2}=\Gamma(q(n)) \sum_{\alpha \geqslant 0} \frac{\alpha !}{\Gamma(q(n)+|\alpha|)}\left|b_{\alpha}\right|^{2} .
\end{gathered}
$$


Exactly as before one shows that $\mathcal{H}_{n, q}$ is a functional Hilbert space of holomorphic functions in $B$ whose reproducing kernel is

$$
\begin{aligned}
K_{n, q}(z, \zeta) & =\frac{1}{\Gamma(q(n))} \sum_{\alpha \geqslant 0} \frac{1}{\alpha !} \Gamma(q(n)+|\alpha|) z^{\alpha \bar{\zeta}^{\alpha}} \\
& =\frac{1}{\Gamma(q(n))} \sum_{m=0}^{\infty} \frac{1}{m !} \Gamma(q(n)+m)\left(\sum_{|\alpha|=m} \frac{m !}{\alpha !} z^{\alpha \bar{\zeta}^{\alpha}}\right) \\
& =\sum_{m=0}^{\infty} \frac{\Gamma(q(n)+m)}{m ! \Gamma(q(n))}\langle z, \zeta\rangle^{m},
\end{aligned}
$$

and, therefore,

$$
K_{n, q}(z, \zeta)=(1-\langle z, \zeta\rangle)^{-q(n)} ; \quad z, \zeta \in B .
$$

For $q(n) \geqslant n$ (i.e., for $q \geqslant 2 n /(n+1))$ the norm (3.4) of $\mathcal{H}_{n, q}$ can be realized as

$$
\|g\|_{n, q}^{2}=\frac{1}{\pi^{n}} \frac{\Gamma(q(n))}{\Gamma(q(n)-n)} \int_{B}|g(z)|^{2}\left(1-|z|^{2}\right)^{q(n)-(n+1)} d \sigma(z), \quad q(n)>n,
$$

and

$$
\|g\|_{n, q}^{2}=\frac{(n-1) !}{2 \pi^{n}} \int_{S}|g(z)|^{2} d s(z), \quad q(n)=n,
$$

where in the last integral, $g$ stands for the nontangential boundary values of the holomorphic function $g(z)$ in $B$. These representations follow from (2.4)-(2.5) and (3.4). It thus follows that $\mathcal{H}_{n, q}$ for $q(n)=n$ is the ordinary "Hardy-Szegö space" with the "Cauchy-Szegö kernel" $(1-\langle z, \zeta\rangle)^{-n}[6$, p. 38], and, for $q(n)=n+1$, $\mathcal{H}_{n, q}$ is the ordinary "Bergman-space" with the "Bergman-kernel" $(1-\langle z, \zeta\rangle)^{-(n+1)}$ $\left[6\right.$, p. 36]. The space $\mathcal{H}_{n, q}$ with $q(n)>n$ is a natural generalization of the Bergman space and was first studied by Selberg [9] for more general homogeneous domains. For $0<q(n)<n$, the norm of $\mathcal{H}_{n, q}$ does not admit such a simple integral representation, a fact which is not crucial, for we shall only use (3.4) as the norm of $g \in \mathcal{H}_{n, q}, q>0$.

The Dirichlet-space $\mathscr{D}_{n, q}$ and the Bergman-Selberg space $\mathcal{H}_{n, q}$ are intimately related. In fact, from (3.3) and (3.5) we have

$$
K_{n, q}(z, \zeta)=\exp \left[k_{n, q}(z, \zeta)\right] ; \quad q>0, z, \zeta \in B,
$$

which in some sense means that $\mathcal{H}_{n, q}$ is the exponentiation of $\mathscr{\mathscr { D }}_{n, q}$. The following theorem is even more striking.

TheOREM 1. Let $f \in \mathscr{O}_{n, q}, q>0$. Then $\exp f \in \mathcal{H}_{n, q}$ and

$$
\|\exp f\|_{n, q}^{2} \leqslant \exp \|f\|_{D_{n, q}}^{2} .
$$

Equality holds if and only if $f$ is of the form $f=k_{n, q}(\cdot, \zeta)$ for some $\zeta \in B$, that is

$$
f(z)=-q(n) \log (1-\langle z, \zeta\rangle), \quad z \in B,
$$

for some $\zeta \in B$. 
The inequality and the sufficient condition in the equality statement of this theorem constitute a simple consequence of a more abstract theorem in the general theory of reproducing kernels (see Burbea [2] for details). The essence, therefore, of this theorem is in the necessity of the equality statement. In the special case that $n=1$ and $q \geqslant 1$, a proof of Theorem 1 was given by Saitoh [7]. His proof, however, is difficult and rather involved. In [2], Burbea has extended the result of Saitoh to cover the case of $0<q<1$ with a proof that is substantially easier and shorter than that of [7]. The method of proof in Burbea [2] conceals in it the main ingredient for establishing the present Theorem 1 . This theorem will be proved as a special case of a more general result, valid for entire functions and functions holomorphic in $B$ or $P$.

While the previous assertion relates two functional Hilbert spaces whose reproducing kernels are related via the exponential function, the next theorem gives a sharp norm inequality in a functional Hilbert space whose reproducing kernel is the ordinary product of reproducing kernels of other functional Hilbert spaces. Other generalizations are possible. However, for clarity we shall confine ourselves to the following case: For $q_{j}>0, j=1, \ldots, m$, we write

$$
q_{j}(n) \equiv(n+1) q_{j} / 2, \quad j=1, \ldots, m .
$$

THEOREM 2. Let $q_{j}>0$ and $g_{j} \in \mathcal{H}_{n, q_{j}}, j=1, \ldots, m$. Then $\prod_{j=1}^{m} g_{j} \in \mathcal{H}_{n, q_{1}+\cdots+q_{m}}$ and

$$
\left\|\prod_{j=1}^{m} g_{j}\right\|_{n, q_{1}+\cdots+q_{m}} \leqslant \prod_{j=1}^{m}\left\|g_{j}\right\|_{n, q_{j}} .
$$

Equality holds if and only if either $\prod_{j=1}^{m} g_{j}=0$ or each $g_{j}(1 \leqslant j \leqslant m)$ is of the form

$$
g_{j}(z)=C_{j}(1-\langle z, \zeta\rangle)^{-q_{j}(n)} ; \quad z \in B, j=1, \ldots, m,
$$

for some (common) $\zeta \in B$ and some nonzero constants $C_{j}(1 \leqslant j \leqslant m)$.

Again, this sharp inequality will be obtained as a special case of a more general result. The last two theorems when combined yield the following interesting sharp inequality. and

THEOREM 3. Let $q_{j}>0$ and $f_{j} \in \mathscr{Q}_{n, q_{j}}, j=1, \ldots, m$. Then $\exp \sum_{j=1}^{m} f_{j} \in \mathcal{H}_{n, \Sigma_{j=1}^{m} q_{j}}$

$$
\left\|\exp \sum_{j=1}^{m} f_{j}\right\|_{n, \Sigma_{j=1}^{m} q_{j}}^{2} \leqslant \exp \sum_{j=1}^{m}\left\|f_{j}\right\|_{\mathcal{D}_{n, q_{j}}}^{2} .
$$

Equality holds if and only if each $f_{j}(1 \leqslant j \leqslant m)$ is of the form

$$
f_{j}(z)=-q_{j}(n) \log (1-\langle z, \zeta\rangle) ; \quad z \in B, j=1, \ldots, m,
$$

for some (common) $\zeta \in B$.

4. Fischer spaces. For fixed $p, q>0$ the " $(p, q)$-Fischer space" is defined by

$$
\mathscr{F}_{p, q} \equiv \mathscr{F}_{p, q}(n)=\left\{f \in H\left(\mathbf{C}^{n}\right):\|f\|_{\mathscr{F}_{p, q}}<\infty\right\}
$$


where

$$
\|f\|_{\mathscr{F}_{p, q}}^{2}=\frac{q^{p+n-1} \Gamma(n)}{\Gamma(p+n-1)} \frac{1}{\pi^{n}} \int_{\mathbf{C}^{n}}|f(z)|^{2}|z|^{2(p-1)} e^{-q|z|^{2}} d \sigma(z) .
$$

Let $f \in \mathscr{F}_{p, q}$ so that

$$
f(z)=\sum_{\alpha} a_{\alpha} z^{\alpha}
$$

Using integration in polar coordinates [6, p. 13] and (2.5) we obtain

$$
\begin{aligned}
\frac{1}{\pi^{n}} \int_{\mathbf{C}^{n}}|f(z)|^{2}|z|^{2(p-1)} e^{-q|z|^{2}} d \sigma(z) & \\
& =2 \sum_{\alpha}\left|a_{\alpha}\right|^{2} \frac{\alpha !}{\Gamma(|\alpha|+n)} \int_{0}^{\infty} r^{2(n+|\alpha|+p-2)} e^{-q r^{2}} r d r \\
& =q^{-(p+n-1)} \sum_{\alpha}\left|a_{\alpha}\right|^{2} \frac{\alpha !}{q^{|\alpha|}} \frac{\Gamma(|\alpha|+p+n-1)}{\Gamma(|\alpha|+n)}
\end{aligned}
$$

and, therefore,

$$
\|f\|_{\mathscr{F}_{p, q}}^{2}=\frac{\Gamma(n)}{\Gamma(p+n-1)} \sum_{\alpha} \frac{\alpha !}{q^{|\alpha|}} \frac{\Gamma(|\alpha|+p+n-1)}{\Gamma(|\alpha|+n)}\left|a_{\alpha}\right|^{2} .
$$

As before, (4.1) shows that $\mathscr{F}_{p, q}$ is a functional Hilbert space of entire functions whose reproducing kernel is

$$
\begin{aligned}
k_{p, q}(z, \zeta) & =\frac{\Gamma(p+n-1)}{\Gamma(n)} \sum_{\alpha} \frac{q^{|\alpha|} \Gamma(|\alpha|+n)}{\Gamma(|\alpha|+p+n-1)} \frac{1}{\alpha !} z^{\alpha \bar{\zeta}^{\alpha}} \\
& =\frac{\Gamma(p+n-1)}{\Gamma(n)} \sum_{m=0}^{\infty} \frac{1}{m !} \frac{q^{m} \Gamma(m+n)}{\Gamma(m+p+n-1)}\left(\sum_{|\alpha|=m} \frac{m !}{\alpha !} z^{\alpha \bar{\zeta} \alpha}\right) \\
& =\frac{\Gamma(p+n-1)}{\Gamma(n)} \sum_{m=0}^{\infty} \frac{1}{m !} \frac{\Gamma(m+n)}{\Gamma(m+p+n-1)}\{q\langle z, \zeta\rangle\}^{m}
\end{aligned}
$$

Therefore

$$
k_{p, q}(z, \zeta)={ }_{1} F_{1}(n ; p+n-1 ; q\langle z, \zeta\rangle)
$$

where ${ }_{1} F_{1}(\alpha ; \beta ; x)$ is the well-known confluent hypergeometric function [10, p. 35]. In particular,

$$
k_{1, q}(z, \zeta)={ }_{1} F_{1}(n ; n ; q\langle z, \zeta\rangle)=e^{q\langle z, \zeta\rangle} .
$$

The space $\mathscr{F}_{1,1} \equiv \mathscr{F}_{1,1}(n)$ is the ordinary Fischer space, a space studied by several authors (cf. [1] and [5]) from various points of view.

Closely related to $\mathscr{F}_{p, q} \equiv \mathscr{F}_{p, q}(n)$ is another functional Hilbert space $\mathcal{E}_{p, q} \equiv \mathcal{E}_{p, q}(n)$ of entire functions $g$ in $\mathbf{C}^{n}$ with norm $\|g\|_{p, q}$ and whose reproducing kernel is

$$
K_{p, q}(z, \zeta)=e^{-1} \exp \left\{{ }_{1} F_{1}(n ; p+n-1 ; q\langle z, \zeta\rangle)\right\} .
$$

In particular,

$$
K_{1, q}(z, \zeta)=e^{-1} \exp \{\exp (q\langle z, \zeta\rangle)\}
$$


For clarity of the exposition we shall here confine ourselves to the case $p=1$. The more general case is deferred to $\$$. We first introduce the "Stirling numbers of the second kind":

$$
s_{k}=\sum_{m=0}^{\infty} \frac{m^{k}}{m !}, \quad k=0,1, \ldots ;
$$

thus, $s_{0}=e, s_{1}=e, s_{2}=2 e, s_{3}=5 e$ and so on. The space $\varepsilon_{1, q}$ is defined by

$$
\varepsilon_{1, q}=\left\{g \in H\left(\mathbf{C}^{n}\right):\|g\|_{1, q}<\infty\right\},
$$

where, for $g \in H\left(\mathbf{C}^{n}\right)$ with

$$
\begin{gathered}
g(z)=\sum_{\alpha \geqslant 0} b_{\alpha} z^{\alpha}, \quad z \in \mathbf{C}^{n}, \\
\|g\|_{1, q}^{2}=e \sum_{\alpha \geqslant 0} \frac{\alpha !}{q^{|\alpha|} s_{|\alpha|}}\left|a_{\alpha}\right|^{2}
\end{gathered}
$$

The expression (4.5) for the reproducing kernel of $\mathcal{E}_{1, q}$ may be verified with the aid of (4.6) and (4.7) as follows:

$$
\begin{aligned}
K_{1, q}(z, \zeta) & =e^{-1} \sum_{\alpha \geqslant 0} q^{|\alpha|} s_{|\alpha|} \frac{1}{\alpha !} z^{\alpha \bar{\zeta}^{\alpha}}=e^{-1} \sum_{\alpha \geqslant 0}\left(\sum_{k=|\alpha|} q^{k} s_{k}\right) \frac{1}{\alpha !} z^{\alpha \bar{\zeta} \alpha} \\
= & e^{-1} \sum_{k=0}^{\infty} \frac{1}{k !} q^{k} s_{k}\left(\sum_{|\alpha|=k} \frac{k !}{\alpha !} z^{\alpha \bar{\zeta}}\right)=e^{-1} \sum_{k=0}^{\infty} \frac{1}{k !} s_{k} q^{k}\langle z, \zeta\rangle^{k} \\
= & e^{-1} \sum_{k=0}^{\infty} \frac{1}{k !}\left(\sum_{m=0}^{\infty} \frac{m^{k}}{m !}\right) q^{k}\langle z, \zeta\rangle^{k}=e^{-1} \sum_{m=0}^{\infty} \frac{1}{m !}\left(\sum_{k=0}^{\infty} \frac{1}{k !} m^{k} q^{k}\langle z, \zeta\rangle^{k}\right) \\
= & e^{-1} \sum_{m=0}^{\infty} \frac{1}{m !} e^{m q\langle z, \zeta\rangle}
\end{aligned}
$$

and (4.5) follows.

Analogous to Theorems 1 and 2 we have the following theorems.

THEOREM 4. Let $f \in \mathscr{F}_{1, q}(n), q>0$, with $f(0)=0$. Then $\exp f \in \mathcal{E}_{1, q}(n)$ and

$$
\|\exp f\|_{1, q}^{2} \leqslant \exp \|f\|_{\mathscr{F}_{1, q}}^{2} .
$$

Equality holds if and only if $f$ is of the form

$$
f(z)=e^{q\langle z, \zeta\rangle}-1, \quad z \in \mathbf{C}^{n},
$$

for some $\zeta \in \mathbf{C}^{n}$.

TheOrem 5. Let $q_{j}>0$ and $f_{j} \in \mathscr{F}_{1, q_{j}}(n), j=1, \ldots, m$. Then $\Pi_{j=1}^{m} f_{j} \in$ $\mathscr{F}_{1, q_{1}+\cdots+q_{m}}(n)$ and

$$
\left\|\prod_{j=1}^{m} f_{j}\right\|_{1, q_{1}+\cdots+q_{m}} \leqslant \prod_{j=1}^{m}\left\|f_{j}\right\|_{1, q_{j}} ;
$$


equivalently,

$$
\begin{aligned}
\int_{\mathbf{C}^{n}}\left|\prod_{j=1}^{m} f_{j}(z)\right|^{2} e^{-\left(q_{1}+\cdots+q_{m}\right)|z|^{2}} d \sigma(z) & \\
& \leqslant\left(\frac{q_{1} \cdots q_{m}}{q_{1}+\cdots+q_{m}}\right)^{n} \frac{1}{\pi^{n(m-1)}} \prod_{j=1}^{m}\left\{\int_{\mathbf{C}^{n}}\left|f_{j}(z)\right|^{2} e^{-q_{j}|z|^{2}} d \sigma(z)\right\}
\end{aligned}
$$

Equality holds if and only if either $\prod_{j=1}^{m} f_{j}=0$ or each $f_{j}(1 \leqslant j \leqslant m)$ is of the form

$$
f_{j}(z)=C_{j} e^{q_{j}\langle z, \zeta\rangle}, \quad z \in \mathbf{C}^{n},
$$

for some (common) $\zeta \in \mathbf{C}^{n}$ and some nonzero constants $C_{j}(1 \leqslant j \leqslant m)$.

A special case of Theorem 5, namely when $n=1$ and $q_{1}=\cdots=q_{m}=1$, appears also in Saitoh [8]. The proof of Saitoh for this special case is rather difficult and is based on the theory of tensor products of reproducing kernel spaces of entire functions. Also, it appears that the equality statement in [8] contains an error or a misprint. The last two theorems will also be obtained as special cases of more general results on spaces of square summable series.

5. Square summable series spaces. We consider the space $H\left(D_{\rho}\right), \rho=0,1, \infty$. We shall also use the notation of $z \bar{\zeta}=\left(z_{1} \bar{\zeta}_{1}, \ldots, z_{n} \bar{\zeta}_{n}\right)$ for $z, \zeta \in \mathbf{C}^{n}$. By $\mathcal{P}\left(D_{\rho}\right)$ we denote the class of all functions $\phi$ with an expansion

$$
\phi(z \bar{z})=\sum_{\alpha} c_{\alpha}(z \bar{z})^{\alpha} ; \quad c_{\alpha}>0, \alpha \in \mathbf{Z}_{+}^{n}, z \in D_{\rho},
$$

which converges on compacta of $D_{\rho}$, but $\phi(z \bar{z})=\infty$ when $z$ lies on the boundary of $D_{\rho}$. Evidently, for any $\zeta \in D_{\rho}, \phi(\cdot \bar{\zeta}) \in H\left(D_{\rho}\right)$. Associated with any $\phi \in \mathcal{P}\left(D_{\rho}\right)$ we consider the space

$$
\mathcal{H}_{\phi}=\left\{f \in H\left(D_{\rho}\right):\|f\|_{\phi}<\infty\right\}
$$

where, for

$$
\begin{gathered}
f(z)=\sum_{\alpha} a_{\alpha} z^{\alpha} ; \quad f \in H\left(D_{\rho}\right), z \in D_{\rho}, \\
\|f\|_{\phi}^{2}=\sum_{\alpha} c_{\alpha}^{-1}\left|a_{\alpha}\right|^{2} .
\end{gathered}
$$

For $\zeta \in D_{\rho}$, we have

$$
f(\zeta)=\sum_{\alpha} a_{\alpha} \zeta^{\alpha}=\sum_{\alpha} c_{\alpha}^{-1}\left(a_{\alpha} c_{\alpha} \zeta^{\alpha}\right)=(f(\cdot), \phi(\cdot \bar{\zeta}))_{\phi}
$$

and, therefore,

$$
|f(\zeta)| \leqslant\{\phi(\zeta \bar{\zeta})\}^{1 / 2}\|f\|_{\phi} ; \quad f \in \mathcal{H}_{\phi}, \zeta \in D_{\rho} .
$$

This shows that $\mathcal{H}_{\phi}$ is a functional Hilbert space of holomorphic functions in $D_{\rho}$ with the reproducing kernel

$$
k_{\phi}(z, \zeta)=\phi(z \bar{\zeta}) ; \quad z, \zeta \in D_{\rho}
$$


The norm of $\mathcal{H}_{\phi}$ is given by (5.2) which also shows that $\left\{\phi_{\alpha}\right\}_{\alpha \geqslant 0}$ with $\phi_{\alpha}(z)=\sqrt{c_{\alpha}} z^{\alpha}$ is an orthonormal basis for $\mathcal{H}_{\phi}$.

In the definition of $\phi \in \mathcal{P}\left(D_{\rho}\right)$ we may allow some of the coefficients $c_{\alpha}$ to equal zero. This corresponds to $\phi(z \bar{\zeta})$ being the reproducing kernel of a functional Hilbert space of holomorphic functions in $D_{\rho}$ with certain (mixed) partial derivatives vanishing at the origin. For example, if $c_{0}=0$ then $\phi(0)=0$ and $\mathcal{H}_{\phi}=\left\{f \in H\left(D_{\rho}\right)\right.$ : $\left.f(0)=0,\|f\|_{\phi}<\infty\right\}$. Such a $\phi$ is said to belong to class $\mathscr{P}_{0}\left(D_{\rho}\right)$.

We note that $\phi, \psi \in \mathcal{P}\left(D_{\rho}\right)$ implies $\phi \psi \in \mathcal{P}\left(D_{\rho}\right)$. With this observation we state our basic lemma.

LEMMA 1. Let $\phi, \psi \in \mathcal{P}\left(D_{\rho}\right)$ and $f \in \mathcal{H}_{\phi}, g \in \mathcal{H}_{\psi}$. Then $f g \in \mathcal{H}_{\phi \psi}$ and

$$
\|f g\|_{\phi \psi} \leqslant\|f\|_{\phi}\|g\|_{\psi} \text {. }
$$

Equality holds if and only if either $f g=0$ or $f$ and $g$ are of the forms $f(z)=C_{1} \phi(z \bar{\zeta})$, $g(z)=C_{2} \psi(z \bar{\zeta}) ; z \in D_{\rho}$, for some (common) $\zeta \in D_{\rho}$ and some nonzero constants $C_{1}$ and $C_{2}$.

Proof. We assume that $\phi, f$ and $\|f\|_{\phi}$ are as in (5.1)-(5.2). The corresponding quantities for $\mathcal{H}_{\psi}$ will be given by

$$
\begin{gathered}
\psi(z)=\sum_{\alpha} d_{\alpha} z^{\alpha} ; \quad d_{\alpha}>0, \alpha \in \mathbf{Z}_{+}^{n}, z \in D_{\rho}, \\
g(z)=\sum_{\alpha} b_{\alpha} z^{\alpha}, \quad z \in D_{\rho},
\end{gathered}
$$

and

$$
\|g\|_{\psi}^{2}=\sum_{\alpha} d_{\alpha}^{-1}\left|b_{\alpha}\right|^{2}
$$

Under these circumstances,

$$
\begin{array}{ll}
\phi(z) \psi(z)=\sum_{\alpha} M_{\alpha} z^{\alpha}, & M_{\alpha}=\sum_{\beta \leqslant \alpha} c_{\beta} d_{\alpha-\beta}, \\
f(z) g(z)=\sum_{\alpha} A_{\alpha} z^{\alpha}, & A_{\alpha}=\sum_{\beta \leqslant \alpha} a_{\beta} b_{\alpha-\beta},
\end{array}
$$

and

$$
\|f g\|_{\phi \psi}^{2}=\sum_{\alpha} M_{\alpha}^{-1}\left|A_{\alpha}\right|^{2}
$$

The lemma, therefore, is completely equivalent to the following sharp inequality:

$$
\sum_{\alpha} M_{\alpha}^{-1}\left|A_{\alpha}\right|^{2} \leqslant\left(\sum_{\alpha} c_{\alpha}^{-1}\left|a_{\alpha}\right|^{2}\right)\left(\sum_{\alpha} d_{\alpha}^{-1}\left|b_{\alpha}\right|^{2}\right)
$$

with equality if and only if either (i) $a_{\alpha}=0$ or $b_{\alpha}=0$ for all $\alpha \in \mathbf{Z}_{+}^{n}$, or (ii) $a_{\alpha}=C_{1} c_{\alpha} \bar{\zeta}^{\alpha}$ and $b_{\alpha}=C_{2} d_{\alpha} \bar{\zeta}^{\alpha}$ for all $\alpha \in \mathbf{Z}_{+}^{n}$, for some $\zeta \in D_{\rho}$ and for some 
nonzero constants $C_{1}$ and $C_{2}$. In order to prove this inequality we let $r=\left(r_{1}, \ldots, r_{n}\right)$ $\in[0,1)^{n}$ and introduce

$$
\begin{gathered}
M(r)=\sum_{\alpha} M_{\alpha}^{-1}\left|A_{\alpha}\right|^{2} r^{\alpha}, \quad C(r)=\sum_{\alpha} c_{\alpha}^{-1}\left|a_{\alpha}\right|^{2} r^{\alpha}, \\
D(r)=\sum_{\alpha} d_{\alpha}^{-1}\left|b_{\alpha}\right|^{2} r^{\alpha} .
\end{gathered}
$$

By the Cauchy-Schwarz inequality,

$$
\begin{aligned}
\left|A_{\alpha}\right|^{2} & =\left|\sum_{\beta \leqslant \alpha} a_{\beta} b_{\alpha-\beta}\right|^{2}=\left|\sum_{\beta \leqslant \alpha} \frac{a_{\beta} b_{\alpha-\beta}}{\left(c_{\beta} d_{\alpha-\beta}\right)^{1 / 2}}\left(c_{\beta} d_{\alpha-\beta}\right)^{1 / 2}\right|^{2} \\
& \leqslant\left(\sum_{\beta \leqslant \alpha} \frac{\left|a_{\beta}\right|^{2}}{c_{\beta}} \frac{\left|b_{\alpha-\beta}\right|^{2}}{d_{\alpha-\beta}}\right)\left(\sum_{\beta \leqslant \alpha} c_{\beta} d_{\alpha-\beta}\right)=\left(\sum_{\beta \leqslant \alpha} \frac{\left|a_{\beta}\right|^{2}}{c_{\beta}} \frac{\left|b_{\alpha-\beta}\right|^{2}}{d_{\alpha-\beta}}\right) M_{\alpha},
\end{aligned}
$$

and so

$$
M_{\alpha}^{-1}\left|A_{\alpha}\right|^{2} \leqslant \sum_{\beta \leqslant \alpha} \frac{\left|a_{\beta}\right|^{2}}{c_{\beta}} \cdot \frac{\left|b_{\alpha-\beta}\right|^{2}}{d_{\alpha-\beta}}, \quad \alpha \in \mathbf{Z}_{+}^{n} .
$$

This shows that

$$
M(r) \leqslant C(r) D(r), \quad r=\left(r_{1}, \ldots, r_{n}\right) \in[0,1)^{n},
$$

as formal power series. Letting $r=\left(r_{1}, \ldots, r_{n}\right) \rightarrow 1^{-}=\left(1^{-}, \ldots, 1^{-}\right)$in (5.6), inequality (5.4) is obtained. In view of (5.4)-(5.6) and the fact that $M(0)=C(0) D(0)$, equality in (5.4) holds if and only if $M(r)=C(r) D(r)$ for each $r=\left(r_{1}, \ldots, r_{n}\right) \in[0,1)^{n}$, which is equivalent to having equality in (5.5) for every $\alpha \in \mathbf{Z}_{+}^{n}$. This is, obviously, equivalent to an existence of $\lambda_{\alpha} \in \mathbf{C}$ so that

$$
a_{\beta} b_{\alpha-\beta}=\lambda_{\alpha} c_{\beta} d_{\alpha-\beta} ; \quad \beta \leqslant \alpha, \alpha \in \mathbf{Z}_{+}^{n} .
$$

Putting $\beta=0$ and $\beta=\alpha$ in (5.7) results in

$$
\lambda_{\alpha} c_{0} d_{\alpha}=a_{0} b_{\alpha}, \quad \lambda_{\alpha} c_{\alpha} d_{0}=a_{\alpha} b_{0} ; \quad \alpha \in \mathbf{Z}_{+}^{n} .
$$

On the other hand, summing up (5.7) from $\beta=0$ through $\beta=\alpha$ yields

$$
A_{\alpha}=\lambda_{\alpha} M_{\alpha}, \quad \alpha \in \mathbf{Z}_{+}^{n} .
$$

If $a_{0} b_{0}=0$, then by (5.8), $\lambda_{\alpha}=0$ for all $\alpha \in \mathbf{Z}_{+}^{n}$. Therefore, by (5.9), $A_{\alpha}=0$ for all $\alpha \in \mathbf{Z}_{+}^{n}$ which means $a_{\alpha}=0$ or $b_{\alpha}=0$ for all $\alpha \in \mathbf{Z}_{+}^{n}$. This covers item (i) of the equality statement. We now assume that $a_{0} b_{0} \neq 0$ and define

$$
C_{1}=a_{0} c_{0}^{-1}, \quad C_{2}=b_{0} d_{0}^{-1} \text {. }
$$

We also define

$$
\zeta_{k}=c_{0} \bar{a}_{e_{k}}\left(c_{e_{k}} \bar{a}_{0}\right)^{-1}, \quad 1 \leqslant k \leqslant n .
$$

It follows from (5.8) that also

$$
\zeta_{k}=d_{0} \bar{b}_{e_{k}}\left(d_{e_{k}} \bar{b}_{0}\right)^{-1}, \quad 1 \leqslant k \leqslant n .
$$


Clearly, $C_{1}, C_{2} \neq 0$. From (5.7)-(5.10) we have

$$
\begin{gathered}
b_{\alpha}=C_{2} C_{1}^{-1}\left(a_{\alpha} / c_{\alpha}\right) d_{\alpha}, \quad \alpha \in \mathbf{Z}_{+}^{n}, \\
a_{0} b_{\alpha}+\sum_{0<\beta<\alpha} a_{\beta} b_{\alpha-\beta}+a_{\alpha} b_{0}=\frac{a_{\alpha} b_{0}}{c_{\alpha} d_{0}} \sum_{\beta \leqslant \alpha} c_{\beta} d_{\alpha-\beta}, \quad \alpha \in \mathbf{Z}_{+}^{n}-\{0\},
\end{gathered}
$$

and, therefore,

$$
\begin{aligned}
a_{\alpha} b_{0}\left(c_{\alpha} d_{0}+c_{0} d_{\alpha}\right)+c_{\alpha} d_{0} \sum_{0<\beta<\alpha} a_{\beta} b_{\alpha-\beta}=a_{\alpha} b_{0} \sum_{\beta \leq \alpha} c_{\beta} d_{\alpha-\beta}, & \\
\alpha & \in \mathbf{Z}_{+}^{n}-\{0\} .
\end{aligned}
$$

We use induction on the weight $|\alpha|$ to show that with $\zeta=\left(\zeta_{1}, \ldots, \zeta_{n}\right)$,

$$
a_{\alpha}=C_{1} c_{\alpha} \bar{\zeta}^{\alpha}, \quad b_{\alpha}=C_{2} d_{\alpha} \bar{\zeta}^{\alpha} ; \quad \alpha \in \mathbf{Z}_{+}^{n} .
$$

Clearly, by (5.10)-(5.13), (5.15) is true for $|\alpha|=0$ and $|\alpha|=1$. Assuming (5.15) is true for $\alpha, 0 \leqslant|\alpha| \leqslant m-1, m \geqslant 2$, we find by (5.14) that for $|\alpha|=m$,

$$
a_{\alpha} b_{0}\left(c_{\alpha} d_{0}+c_{0} d_{\alpha}\right)+c_{\alpha} d_{0} C_{1} C_{2} \bar{\zeta}^{\alpha} \sum_{0<\beta<\alpha} c_{\beta} d_{\alpha-\beta}=a_{\alpha} b_{0} \sum_{\beta \leqslant \alpha} c_{\beta} d_{\alpha-\beta},
$$

and so, by (5.10),

$$
a_{\alpha} b_{0} \sum_{0<\beta<\alpha} c_{\beta} d_{\alpha-\beta}=C_{1} c_{\alpha} \bar{\zeta}^{\alpha} b_{0} \sum_{0<\beta<\alpha} c_{\beta} d_{\alpha-\beta}, \quad|\alpha|=m \geqslant 2 .
$$

This shows that $a_{\alpha}=C_{1} c_{\alpha} \bar{\zeta}^{\alpha}$, and, by (5.13), also $b_{\alpha}=C_{2} d_{\alpha} \bar{\zeta}^{\alpha}$ and (5.15) is proved. Finally, $\zeta$ must lie in $D_{\rho}$ because, for example, the value of the first factor on the right-hand side of (5.4) for the solution in (5.15) is $\left|C_{1}\right|^{2} \phi(\zeta \bar{\zeta})$ and, since the domain of convergence of $\phi(\zeta \bar{\zeta})$ is $D_{\rho}(\rho=0,1, \infty)$, the assertion follows. This concludes the proof.

An immediate consequence of this lemma is

TheOREM 6. Let $\phi_{j} \in \mathcal{P}\left(D_{\rho}\right)$ and $f_{j} \in \mathcal{H}_{\phi_{j}}, j=1, \ldots$, m. Then $\prod_{j=1}^{m} f_{j} \in \mathcal{H}_{\phi_{1} \cdots \phi_{m}}$ and

$$
\left\|\prod_{j=1}^{m} f_{j}\right\|_{\phi_{1} \cdots \phi_{m}} \leqslant \prod_{j=1}^{m}\left\|f_{j}\right\|_{\phi_{j}}
$$

with equality if and only if either $\prod_{j=1}^{m} f_{j}=0$ or each $f_{j}$ is of the form $f_{j}(z)=C_{j} \phi_{j}(z \bar{\zeta})$, $z \in D_{\rho}$, for some (common) $\zeta \in D_{\rho}$ and some nonzero constants $C_{j}(1 \leqslant j \leqslant m)$.

An analogous sharp inequality with respect to the ordinary sum of reproducing kernels is also available. In fact, one can directly verify the following theorem.

TheOREM 7. Let $\phi_{j} \in \mathcal{P}\left(D_{\rho}\right)$ and $f_{j} \in \mathcal{H}_{\phi_{j}}, j=1, \ldots, m$. Then $\sum_{j=1}^{m} f_{j} \in \mathcal{H}_{\Sigma \phi_{j}}$ and

$$
\left\|\sum_{j=1}^{m} f_{j}\right\|_{\Sigma \phi_{j}}^{2} \leqslant \sum_{j=1}^{m}\left\|f_{j}\right\|_{\phi_{j}}^{2}
$$

with equality if and only if each $f_{j}$ is of the form $f_{j}(z)=C \phi_{j}(z \bar{\zeta}), z \in D_{\rho}$, for some (common) $\zeta \in D_{\rho}$ and some constant $C$. 
6. Exponentiation. Let $\alpha \in \mathbf{Z}_{+}^{n}-\{0\}$ and let $\alpha_{k}, 1 \leqslant k \leqslant n$, be a nonzero component of $\alpha$. We then write

$$
\alpha-1_{k}=\left(\alpha_{1}, \ldots, \alpha_{k}-1, \ldots, \alpha_{n}\right) ; \quad \alpha_{k} \geqslant 1,1 \leqslant k \leqslant n .
$$

Given an $f \in H\left(D_{\rho}\right), f(0)=0$, with

$$
f(z)=\sum_{\alpha \geqslant 1} a_{\alpha} z^{\alpha} \quad(f(0)=0)
$$

we consider its exponential transform

$$
g(z)=\exp \{f(z)\}=\sum_{\alpha \geq 0} b_{\alpha} z^{\alpha},
$$

and hence $b_{0}=1$. For an arbitrary $k, 1 \leqslant k \leqslant n,(6.2)$ shows that

$$
\left(\partial / \partial z_{k}\right) g(z)=g(z)\left(\partial / \partial z_{k}\right) f(z)
$$

and therefore, by (6.1)-(6.2),

$$
\sum_{\alpha_{k} \geqslant 1} \alpha_{k} b_{\alpha} z^{\alpha-1_{k}}=\left(\sum_{\alpha \geqslant 0} b_{\alpha} z^{\alpha}\right)\left(\sum_{\alpha_{k} \geqslant 1} \alpha_{k} a_{\alpha} z^{\alpha-1_{k}}\right)=\sum_{\alpha_{k} \geqslant 1}\left(\sum_{\beta \leqslant \alpha} \beta_{k} a_{\beta} b_{\alpha-\beta}\right) z^{\alpha-1_{k}} .
$$

This shows that

$$
b_{\alpha}=\frac{1}{\alpha_{k}} \sum_{\beta \leqslant \alpha} \beta_{k} a_{\beta} b_{\alpha-\beta} ; \quad \alpha_{k} \geqslant 1\left(b_{0}=1\right),
$$

a formula which is independent of $k, 1 \leqslant k \leqslant n$, with $\alpha_{k} \geqslant 1$.

Let $\phi \in \mathscr{P}_{0}\left(D_{\rho}\right)$ so that

$$
\phi(z \bar{z})=\sum_{\alpha \geqslant 1} c_{\alpha}(z \bar{z})^{\alpha} ; \quad c_{\alpha}>0, \alpha \in \mathbf{Z}_{+}^{n}-\{0\}, z \in D_{\rho}(\phi(0)=0),
$$

and consider its exponential transform

$$
\psi(z \bar{z})=\exp \{\phi(z \bar{z})\}, \quad z \in D_{\rho} .
$$

Clearly, $\psi \in \mathcal{P}\left(D_{\rho}\right)$ and, in view of (6.3), the coefficients of its expansion

$$
\psi(z \bar{z})=\sum_{\alpha \geqslant 0} d_{\alpha}(z \bar{z})^{\alpha}
$$

satisfy

$$
d_{\alpha}=\frac{1}{\alpha_{k}} \sum_{\beta \leqslant \alpha} \beta_{k} c_{\beta} d_{\alpha-\beta} ; \quad \alpha_{k} \geqslant 1\left(d_{0}=1\right) .
$$

This shows that $d_{e_{k}}=c_{e_{k}}(1 \leqslant k \leqslant n)$ and, moreover,

LEMMA 2. Let the notation of (6.4)-(6.7) apply. Then $d_{\alpha}=1$ for $\alpha=0, d_{\alpha}=c_{\alpha}$ for $|\alpha|=1$ and $d_{\alpha}>c_{\alpha}$ for $|\alpha| \geqslant 2$.

Proof. The lemma is obvious for $|\alpha| \leqslant 1$. For $|\alpha| \geqslant 2$ we have, by (6.7),

$$
d_{\alpha}=c_{\alpha}+\frac{1}{\alpha_{k}} \sum_{\beta<\alpha} \beta_{k} c_{\beta} d_{\alpha-\beta}, \quad \alpha_{k} \geqslant 1(1 \leqslant k \leqslant n) .
$$


In particular, since $d_{\alpha}>0$ for any $\alpha \geqslant 0$ and since the above sum contains the $\beta=e_{k}$ term we have

$$
d_{\alpha} \geqslant c_{\alpha}+\left(1 / \alpha_{k}\right) c_{e_{k}} d_{\alpha-e_{k}}>c_{\alpha},
$$

and the lemma follows.

With the above notation, we may consider the functional Hilbert space

$$
\mathcal{H}_{\phi}^{0}=\left\{f \in H\left(D_{\rho}\right): f(0)=0,\|f\|_{\phi}<\infty\right\}
$$

where for $f \in H\left(D_{\rho}\right), f(0)=0$, given as in (6.1),

$$
\|f\|_{\phi}^{2}=\sum_{\alpha \geqslant 1} c_{\alpha}^{-1}\left|a_{\alpha}\right|^{2}
$$

This space has

$$
k_{\phi}(z, \zeta)=\phi(z \bar{\zeta})=\sum_{\alpha \geqslant 1} c_{\alpha}(z \bar{\zeta})^{\alpha} ; \quad z, \zeta \in D_{\rho},
$$

as its reproducing kernel. Also, $\left\{\phi_{\alpha}\right\}_{\alpha \geqslant 1}$ with $\phi_{\alpha}(z)=\sqrt{c_{\alpha}} z^{\alpha}$ is an orthonormal basis for $\mathcal{H}_{\phi}^{0}$.

The new function $\psi$ of (6.5) determines another functional Hilbert space $\mathcal{K}_{\psi}$ of functions $g$,

$$
g(z)=\sum_{\alpha \geq 0} b_{\alpha} z^{\alpha}, \quad z \in D_{\rho},
$$

holomorphic in $D_{\rho}$, with $\|g\|_{\psi}<\infty$ where, in view of (6.6)-(6.7),

$$
\|g\|_{\psi}^{2}=\sum_{\alpha \geqslant 0} d_{\alpha}^{-1}\left|b_{\alpha}\right|^{2}
$$

As before, this space has

$$
k_{\psi}(z, \zeta)=\psi(z \bar{\zeta})=\exp \{\phi(z \bar{\zeta})\}
$$

as its reproducing kernel.

The main theorem in this direction is

TheOREM 8. Let $\phi \in \mathscr{P}_{0}\left(D_{\rho}\right)$ and thus $\psi=\exp \phi \in \mathscr{P}\left(D_{\rho}\right)$. Assume $f \in \mathcal{H}_{\phi}^{0}$; then $\exp f \in \mathcal{H}_{\psi}$ and

$$
\|\exp f\|_{\psi}^{2} \leqslant \exp \|f\|_{\phi}^{2} .
$$

Equality holds if and only if $f$ is of the form $f(z)=k_{\phi}(z, \zeta)=\phi(z \bar{\zeta}), z \in D_{\rho}$, for some $\zeta \in D_{\rho}$.

This theorem is completely equivalent to the following lemma.

LEMMA 3. Let the notation of (6.1)-(6.10) apply. Then

$$
\sum_{\alpha \geqslant 0} d_{\alpha}^{-1}\left|b_{\alpha}\right|^{2} \leqslant \exp \left\{\sum_{\alpha \geqslant 1} c_{\alpha}^{-1}\left|a_{\alpha}\right|^{2}\right\}
$$

if the right-hand side is finite. Equality holds if and only if $a_{\alpha}=c_{\alpha} \bar{\zeta}^{\alpha}(\alpha \geqslant 1)$ and $b_{\alpha}=d_{\alpha} \bar{\zeta}^{\alpha}(\alpha \geqslant 0)$ for some (common) $\zeta \in D_{\rho}$. 
Proof. For $r=\left(r_{1}, \ldots, r_{n}\right) \in[0,1)^{n}$, define

$$
A(r)=\sum_{\alpha \geqslant 1} c_{\alpha}^{-1}\left|a_{\alpha}\right|^{2} r^{\alpha}, \quad B(r)=\sum_{\alpha \geqslant 0} d_{\alpha}^{-1}\left|b_{\alpha}\right|^{2} r^{\alpha} .
$$

Let $\alpha \in \mathbf{Z}_{+}^{n}-\{0\}$ with $\alpha_{k} \geqslant 1,1 \leqslant k \leqslant n$. By (6.3), (6.7) and the Cauchy-Schwarz inequality,

$$
\begin{aligned}
\alpha_{k}^{2}\left|b_{\alpha}\right|^{2} & =\left|\sum_{\beta \leqslant \alpha}\left(\frac{\beta_{k}}{c_{\beta} d_{\alpha-\beta}}\right)^{1 / 2} a_{\beta} b_{\alpha-\beta}\left(\beta_{k} c_{\beta} d_{\alpha-\beta}\right)^{1 / 2}\right|^{2} \\
& \leqslant\left(\sum_{\beta \leqslant \alpha} \beta_{k} \frac{\left|a_{\beta}\right|^{2}}{c_{\beta}} \frac{\left|b_{\alpha-\beta}\right|^{2}}{d_{\alpha-\beta}}\right)\left(\sum_{\beta \leqslant \alpha} \beta_{k} c_{\beta} d_{\alpha-\beta}\right) \\
& =\left(\sum_{\beta \leqslant \alpha} \beta_{k} \frac{\left|a_{\beta}\right|^{2}}{c_{\beta}} \frac{\left|b_{\alpha-\beta}\right|^{2}}{d_{\alpha-\beta}}\right) \alpha_{k} d_{\alpha}
\end{aligned}
$$

and thus

$$
\alpha_{k} d_{\alpha}^{-1}\left|b_{\alpha}\right|^{2} \leqslant \sum_{\beta \leqslant \alpha} \beta_{k} \frac{\left|a_{\beta}\right|^{2}}{c_{\beta}} \frac{\left|b_{\alpha-\beta}\right|^{2}}{d_{\alpha-\beta}}
$$

This shows that

$$
\left(\partial / \partial r_{k}\right) B(r) \leqslant B(r)\left(\partial / \partial r_{k}\right) A(r)
$$

or that

$$
\left(\partial / \partial r_{k}\right) \log B(r) \leqslant\left(\partial / \partial r_{k}\right) A(r), \quad 1 \leqslant k \leqslant n,
$$

as a formal power series. Note that if the right-hand side of $(6.11)$ is finite then $A(r)$ converges for $r \in[0,1)^{n}$ and, therefore, by (6.13), $B(r)$ also converges for $r \in[0,1)^{n}$. Let $\delta=\left(\delta_{1}, \ldots, \delta_{n}\right) \in[0,1)^{n}$ and choose a path $r_{k}=r_{k}(t)$, where $r_{k}(t)$ is increasing in $t \in[0,1]$ and $r_{k}(0)=0, r_{k}(1)=\delta_{k}$ for $1 \leqslant k \leqslant n$. Since $A(0)=0$ and $B(0)=1$, it follows from (6.13) that

$$
\log B(\delta)=\int_{0}^{1} \frac{d}{d t} \log B[r(t)] d t \leqslant \int_{0}^{1} \frac{d}{d t} A[r(t)] d t=A(\delta)
$$

and (6.11) follows by letting $\delta \rightarrow 1^{-}$. In view of the last inequality or (6.13), equality in (6.11) holds if and only if for any $r \in[0,1)^{n}$ and any $k, 1 \leqslant k \leqslant n,(6.13)$ is an equality. This is equivalent to having equality in (6.12) for every component $\alpha_{k} \geqslant 1$. This, in turn, is equivalent to an existence of $\lambda_{\alpha} \in \mathbf{C}$ so that

$$
a_{\beta} b_{\alpha-\beta}=\lambda_{\alpha} c_{\beta} d_{\alpha-\beta}, \quad 1 \leqslant \beta \leqslant \alpha .
$$

Putting $\beta=\alpha$ in (6.14) results in $a_{\alpha}=\lambda_{\alpha} c_{\alpha}$. On the other hand summing up (6.14) from all $\beta$ with $|\beta|=1$ through $\beta$ with $\beta=\alpha$, and, using (6.3) and (6.7), yields $b_{\alpha}=\lambda_{\alpha} d_{\alpha}$. Consequently,

$$
a_{\alpha}=c_{\alpha} d_{\alpha}^{-1} b_{\alpha}, \quad \alpha \geqslant 1,
$$

and with (6.3) this also shows

$$
a_{\alpha}\left(d_{\alpha}-c_{\alpha}\right)=c_{\alpha} \frac{1}{\alpha_{k}} \sum_{\beta<\alpha} \beta_{k} a_{\beta} b_{\alpha-\beta}, \quad \alpha_{k} \geqslant 1(1 \leqslant k \leqslant n) .
$$


We define $\zeta \in\left(\zeta_{1}, \ldots, \zeta_{n}\right) \in \mathbf{C}^{n}$ by

$$
\zeta_{k}=\bar{a}_{e_{k}} \bar{c}_{e_{k}}^{-1}, \quad 1 \leqslant k \leqslant n .
$$

We shall show that the only solution of (6.15) subject to (6.3) and (6.7) is

$$
a_{\alpha}=c_{\alpha} \bar{\zeta}^{\alpha} \quad(\alpha \geqslant 1), \quad b_{\alpha}=d_{\alpha} \bar{\zeta}^{\alpha} \quad(\alpha \geqslant 0) .
$$

This will be accomplished by induction on the weight $|\alpha|$. The case of $|\alpha|=0$ is trivial and for $|\alpha|=1,(6.18)$ is true by virtue of the definition (6.17) and (6.15). We assume that (6.18) is true for $\alpha, 1 \leqslant|\alpha|<m$. When $|\alpha|=m$, there is a component $\alpha_{k} \geqslant 1,1 \leqslant k \leqslant n$, of $\alpha$ so that (6.3), (6.7) and (6.16) apply. By (6.16) and the inductive assumption,

$$
a_{\alpha}\left(d_{\alpha}-c_{\alpha}\right)=c_{\alpha} \bar{\zeta}^{\alpha} \frac{1}{\alpha_{k}} \sum_{\beta<\alpha} \beta_{k} c_{\beta} d_{\alpha-\beta} .
$$

In view of (6.7) this may be written as

$$
a_{\alpha}\left(d_{\alpha}-c_{\alpha}\right)=c_{\alpha} \bar{\zeta}^{\alpha}\left(d_{\alpha}-c_{\alpha}\right) \quad(|\alpha|=m \geqslant 1) .
$$

However, by Lemma 1 we have $d_{\alpha}-c_{\alpha}>0$ for $|\alpha|>1$ and, therefore, $a_{\alpha}=c_{\alpha} \bar{\zeta}^{\alpha}$. With this and (6.15), (6.18) follows at once. Finally, the right-hand side of (6.11) for this solution

$$
\exp \left\{\sum_{\alpha \geqslant 1} c_{\alpha}(\zeta \bar{\zeta})^{\alpha}\right\}=\exp \{\phi(\zeta \bar{\zeta})\}
$$

is assumed to be finite. Since $0 \leqslant \phi(\zeta \bar{\zeta})<\infty$ for every $\zeta \in D_{\rho}(\rho=0,1, \infty)$ and $\phi(\zeta \bar{\zeta})=\infty$ when $\zeta$ is on the boundary of $D_{\rho}$, we must have $\zeta \in D_{\rho}$. This concludes the proof.

The last theorem ties in with a more abstract theorem, proved in Burbea [2], in the general theory of reproducing kernels. In fact, let $k=k(\cdot, \cdot)$ be a positive definite kernel on $\Lambda \times \Lambda$ where $\Lambda$ is an arbitrary nonvoid set. As is well known, there exists a uniquely determined functional Hilbert space $H_{k}$ of functions on $\Lambda$ such that $k$ is its reproducing kernel. Also, let $F$ be a nonconstant entire function with nonnegative coefficients. Clearly, $K(\cdot, \cdot)=F[k(\cdot, \cdot)]$, being itself a positive definite kernel on $\Lambda \times \Lambda$, uniquely determines another functional Hilbert space $H_{K}$ of functions on $\Lambda$ such that $K$ is its reproducing kernel. It follows that if $f \in H_{k}$ then $F \circ f \in H_{K}$ and

$$
\|F \circ f\|_{K}^{2} \leqslant F\left(\|f\|_{k}^{2}\right)
$$

and equality holds if $f$ is of the form $f(z)=k(z, \zeta)$ for all $z \in \Lambda$ and some $\zeta \in \Lambda$.

As a particular case of this result we take $\Lambda=D_{\rho}$ and $k=k_{\phi}$ as in (6.9) with $\phi \in \mathscr{P}_{0}\left(D_{\rho}\right)$. Then $H_{k}=\mathcal{H}_{\phi}^{0}$ with norm as in (6.8). We also let

$$
F(t)=\sum_{m=0}^{\infty} \lambda_{m} t^{m} \quad\left(\lambda_{m}>0, m=0,1, \ldots\right),
$$

and hence $\psi \equiv F \circ \phi \in \mathcal{P}\left(D_{\rho}\right), K=K_{\psi}$ and $H_{K}=\mathcal{H}_{\psi}$. Let the expansion of $\psi$ be given as in (6.6). Consequently, if $f \in \mathcal{H}_{\phi}^{0}$ then $F \circ f \in \mathcal{H}_{\psi}^{0}$ and $\|F \circ f\|_{\psi}^{2} \leqslant F\left(\|f\|_{\phi}^{2}\right)$ or

$$
\sum_{\alpha \geqslant 0} d_{\alpha}^{-1}\left|b_{\alpha}\right|^{2} \leqslant F\left(\sum_{\alpha \geqslant 0} c_{\alpha}^{-1}\left|a_{\alpha}\right|^{2}\right)
$$


with equality if $f=\phi(\cdot \bar{\zeta})$ for some $\zeta \in D_{\rho}$ (see also [2]). The inequality of Theorem 8 and (6.11) are obtained when choosing $F(t)=\exp (t)$.

Theorems 6 and 8 when combined yield the following sharp inequality.

TheOREM 9. Let $\phi_{j} \in \mathscr{P}_{0}\left(D_{\rho}\right)$ and, thus, $\psi_{j}=\exp \phi_{j} \in \mathcal{P}\left(D_{\rho}\right), j=1, \ldots, m$. Assume that $f_{j} \in \mathcal{H}_{\phi_{j}}^{0}, j=1, \ldots, m$. Then $\exp \sum_{j=1}^{m} f_{j} \in \mathcal{H}_{\Pi_{j=1}^{m} \psi_{j}}$ and

$$
\left\|\exp \sum_{j=1}^{m} f_{j}\right\|_{\Pi_{j=1}^{m} \psi_{j}}^{2} \leqslant \exp \sum_{j=1}^{m}\left\|f_{j}\right\|_{\phi_{j}}^{2} .
$$

Equality holds if and only if each $f_{j}(1 \leqslant j \leqslant m)$ is of the form $f_{j}(z)=\phi_{j}(z \bar{\zeta}) ; z \in D_{\rho}$, $j=1, \ldots, m$, for some (common) $\zeta \in D_{\rho}$.

7. Applications. Many interesting norm-inequalities are deductible from Theorems 6-9 by specifying the functions of $\mathcal{P}\left(D_{\rho}\right)$ and $D_{\rho}$ with $\rho=0,1, \infty$. For example, Theorems 1-5 are obtained by such a specification. Before so doing, however, several remarks are in order on the class $\mathscr{P}\left(D_{\rho}\right)$ (or $\left.\mathscr{P}_{0}\left(D_{\rho}\right)\right)$ and reproducing kernels $k(\cdot, \cdot)$ on $D_{\rho} \times D_{\rho}$.

We first recall a couple of definitions (see, for example, Donoghue [4]), valid for any domain $D$ in $\mathbf{C}^{n}$. A kernel $k(\cdot, \cdot)$ defined on $D \times D$ is called sesquiholomorphic in $D$ if for any $\zeta \in D, k(\cdot, \zeta)$ and $\overline{k(\zeta, \cdot)}$ are holomorphic in $D$. This means, in view of Hartog's theorem, that the function $F(z, \bar{\zeta}) \equiv k(z, \zeta)$ is holomorphic in both variables $(z, \bar{\zeta}) ;(z, \zeta) \in D \times D$. A kernel $k(\cdot, \cdot)$ which is positive-definite on $D \times D$ and sesquiholomorphic in $D$ is called a Bergman kernel. Such a kernel gives rise to a unique functional Hilbert space $\mathcal{H}(D)$ of functions holomorphic in $D$ for which $k(\cdot, \cdot)$ is its reproducing kernel. Moreover, for any orthonormal basis $\left\{h_{m}\right\}_{m=1}^{\infty}$ of $\mathcal{H}(D)$,

$$
k(z, \zeta)=\sum_{m=1}^{\infty} h_{m}(z) \overline{h_{m}(\zeta)} ; \quad z, \zeta \in D
$$

where the bilinear sum converges absolutely and uniformly on compacta of $D$. In particular, when $D=D_{\rho}(\rho=0,1, \infty)$,

$$
h_{m}(z)=\sum_{\alpha} d_{\alpha}^{(m)} z^{\alpha} ; \quad z \in D_{\rho}, \alpha \in \mathbf{Z}_{+}^{n},
$$

and, therefore,

$$
k(z, \zeta)=\sum_{\alpha, \beta}\left(\sum_{m=1}^{\infty} d_{\alpha}^{(m)} \overline{d_{\beta}^{(m)}}\right) z^{\alpha \bar{\zeta}^{\beta}} ; \quad z, \zeta \in D_{\rho} .
$$

We now further assume that the nonnegative function $k(z, z), z \in D_{\rho}$, depends only on $\left(\left|z_{1}\right|, \ldots,\left|z_{n}\right|\right) \in D_{\rho}$. This implies, in view of (7.1), that

$$
\sum_{m=1}^{\infty} d_{\alpha}^{(m)} \overline{d_{\beta}^{(m)}}=c_{\alpha} \delta_{\alpha \beta}, \quad c_{\alpha} \geqslant 0,
$$

and so

$$
k(z, \zeta)=\sum_{\alpha} c_{\alpha}(z \bar{\zeta})^{\alpha} ; \quad z, \zeta \in D_{\rho}
$$


To avoid degenerate cases we also assume that $k(z, z)=\infty$ for $z$ on the boundary of $D_{\rho}$. This corresponds to requiring that $c_{\alpha}>0$ for infinitely many $\alpha$ in an indexed set $\Lambda$ of $\mathbf{Z}_{+}^{n}$ and thus the function $\phi$ with $\phi(z \bar{z})=k(z, z)$ is in $\mathscr{P}\left(D_{\rho}\right)$. In an obvious way this means that $\mathcal{H}_{\phi}=\mathcal{H}\left(D_{\rho}\right)$ and that the monomials $\left\{z^{\alpha}\right\}$ are orthogonal in $\mathcal{H}_{\phi}$ with $c_{\alpha}^{-1}, \alpha \in \Lambda$, as their moments. The reproducing kernel of $\mathcal{H}_{\phi}$ is then

$$
k(z, \zeta)=\phi(z \bar{\zeta}) ; \quad z, \zeta \in D_{\rho} .
$$

We note that $D_{1} \subset D_{0} \subset D_{\infty}$ (recall that $D_{0}=P, D_{1}=B$ and $D_{\infty}=C^{n}$ ) and that (7.3) means that $\phi(z \bar{z}), z \in D_{\rho}$, depends only on $\left(\left|z_{1}\right|, \ldots,\left|z_{n}\right|\right)$. It is, therefore, natural to also require

$$
\phi(z \bar{z})=F_{1}\left(\left|z_{1}\right|^{2}\right) \cdots F_{n}\left(\left|z_{n}\right|^{2}\right), \quad z=\left(z_{1}, \ldots, z_{n}\right) \in D_{0},
$$

and

$$
\phi(z \bar{z})=F\left(|z|^{2}\right), \quad z \in D_{1},
$$

where $F_{1}, \ldots, F_{n}$ and $F$ are holomorphic functions in the unit disk $B_{1}$ of $\mathbf{C}$ and have this disk as the domain of thier convergence. In the case of $D_{\infty}=\mathrm{C}^{n}$ the two representations in (7.4) and (7.5) are possible, but now the corresponding functions are required to be entire in $\mathbf{C}$. It is now also clear that the corresponding reproducing kernels for the cases of (7.4) and (7.5) (with $D_{\infty}$ included) are now

$$
k(z, \zeta)=F_{1}\left(z_{1} \bar{\zeta}_{1}\right) \cdots F_{n}\left(z_{n} \bar{\zeta}_{n}\right) ; \quad z, \zeta \in D_{\rho}(\dot{\rho}=0, \infty)
$$

$$
k(z, \zeta)=F(\langle z, \zeta\rangle) ; \quad z, \zeta \in D_{\rho}(\rho=1, \infty)
$$

respectively.

We now discuss Theorems 1-5 as special cases of Theorems 6, 8 and 9 and in light of the above remarks.

That Theorem 1 follows from Theorem 8 is quite straightforward. This is accomplished by taking

$$
\phi(z \bar{z})=q(n) \sum_{\alpha \geqslant 1} \frac{(|\alpha|-1) !}{\alpha !}(z \bar{z})^{\alpha}, \quad z \in D_{1}=B,
$$

and thus $\phi \in \mathscr{P}_{0}\left(D_{1}\right)$. Also, $\phi(z \bar{z})=-q(n) \log \left(1-|z|^{2}\right)$ and we are in case (7.7). Further, the space $\mathcal{H}_{\phi}^{0}$ is now $\mathscr{D}_{n, q}, \psi(z \bar{z})=\exp \{\phi(z \bar{z})\}=\left(1-|z|^{2}\right)^{-q(n)}$ and $\mathcal{K}_{\psi}$ is $\mathcal{H}_{n, q}$. Theorem 2 is obtained from Theorem 6 by taking

$$
\phi_{j}(z \bar{z})=\left(1-|z|^{2}\right)^{-q_{j}(n)} ; \quad z \in D_{1}(1 \leqslant j \leqslant m),
$$

and thus $\mathcal{H}_{\phi_{j}}$ is $\mathcal{H}_{n, q_{j}}$. In a similar fashion Theorem 3 follows from Theorem 9.

Theorem 4 follows from Theorem 8 . However, the latter implies the following stronger version of the former.

THEOREM 4*. Let $f \in \mathscr{F}_{p, q}(n), p, q>0$, with $f(0)=0$. Then $\exp f \in \mathcal{E}_{p, q}(n)$ and

$$
\|\exp f\|_{p, q}^{2} \leqslant \exp \|f\|_{\mathscr{F}_{p, q}}^{2} .
$$

Equality holds if and only if $f$ is of the form

$$
f(z)={ }_{1} F_{1}(n ; p+n-1 ; q\langle z, \zeta\rangle)-1
$$

for some $\zeta \in \mathbf{C}^{n}$. 
This follows on taking

$$
\phi(z \bar{z})={ }_{1} F_{1}\left(n ; p+n-1 ; q|z|^{2}\right)-1, \quad z \in \mathbf{C}^{n},
$$

so that $\phi \in \mathscr{P}_{0}\left(D_{\infty}\right)$ and we are in case (7.7). Also, $\mathcal{H}_{\phi}^{0}=\left\{f \in \mathscr{F}_{p, q}(n): f(0)=0\right\}$. Further,

$$
\psi(z \bar{z})=\exp \{\phi(z \bar{z})\}=e^{-1} \exp \left\{{ }_{1} F_{1}\left(n ; p+n-1 ; q|z|^{2}\right)\right\}
$$

and $\mathcal{H}_{\psi}=\mathcal{E}_{p, q}(n)$. This also shows that $K_{p, q}(\cdot, \cdot)$ in (4.4) is indeed the reproducing kernel for $\mathcal{E}_{p, q}(n)$.

A stronger version of Theorem 5 , where $\mathscr{F}_{1, q_{j}}(n)$ is replaced by $\mathscr{F}_{p_{j}, q_{j}}(n), p_{j}, q_{j}>0$ $(j=1, \ldots, m)$, follows from Theorem 6. Note that the reproducing kernels in Theorem 5 are of both cases (7.6) and (7.7). In a similar manner the following version, analogous to Theorem 3 , is at once deduced from Theorem 9 . Let $q_{j}>0$ and $f_{j} \in \mathscr{F}_{1, q_{j}}(n)$ with $f_{j}(0)=0, j=1, \ldots, m$. Then $\exp \Sigma_{j=1}^{m} f_{j} \in H_{\Psi}$ with

$$
\Psi(z \bar{z})=e^{-m} \prod_{j=1}^{m} \exp \left(\exp q_{j}|z|^{2}\right)
$$

and

$$
\left\|\exp \sum_{j=1}^{m} f_{j}\right\|_{\Psi}^{2} \leqslant \exp \sum_{j=1}^{m}\left\|f_{j}\right\|_{\mathscr{F}_{1, q_{j}}}^{2} .
$$

Equality holds if and only if each $f_{j}(1 \leqslant j \leqslant m)$ is of the form

$$
f_{j}(z)=e^{q_{j}\langle z, \zeta\rangle}-1 ; \quad z \in \mathbf{C}^{n}, j=1, \ldots, m,
$$

for some (common) $\zeta \in \mathbf{C}^{n}$.

Other norm-inequalities valid for functions holomorphic in $D_{\rho}$ are obtainable from Theorems 6-9. We shall conclude the present discussion with a polydisk version of Theorem 1 which may also be regarded as a corollary of Theorem 8 . The proof is quite straightforward and therefore omitted.

Let $A(P)$ be the subclass of $H(P)$ consisting of all functions $f$ of the form

$$
\begin{aligned}
f(z)=\sum_{j=1}^{n} f_{j}\left(z_{j}\right) ; \quad z=\left(z_{1}, \ldots, z_{n}\right) \in P & =B_{1}^{n}, \\
& f_{j} \in H\left(B_{1}\right), j=1, \ldots, n .
\end{aligned}
$$

We shall write $\mathbf{q}>0$ for $\mathbf{q}=\left(q_{1}, \ldots, q_{n}\right) ; q_{j}>0, j=1, \ldots, n$, and we introduce the "q-Dirichlet space"

$$
\mathscr{D}_{\mathbf{q}}=\left\{f \in A(P): f_{j}(0)=0,1 \leqslant j \leqslant n,\|f\|_{\mathscr{D}_{\mathbf{q}}}<\infty\right\},
$$

where for $f \in A(P)$ as in (7.8),

$$
\|f\|_{\mathscr{D}_{\mathrm{q}}}^{2}=\frac{1}{\pi} \sum_{j=1}^{n} \frac{1}{q_{j}} \int_{B_{1}}\left|f_{j}^{\prime}(z)\right|^{2} d \sigma(z) .
$$

This is a functional Hilbert space with the reproducing kernel

$$
k_{\mathbf{q}}(z, \zeta)=-\sum_{j=1}^{n} q_{j} \log \left(1-z_{j} \bar{\zeta}_{j}\right) ; \quad z, \zeta \in P
$$


Analogously, we define the "q-Bergman-Selberg space"

$$
\mathcal{H}_{\mathbf{q}}=\left\{g \in H(P):\|g\|_{\mathbf{q}}<\infty\right\},
$$

where for $g \in H(P)$ with $g(z)=\Sigma_{\alpha \geq 0} b_{\alpha} z^{\alpha}, z \in P$,

$$
\|g\|_{q}^{2}=\left(\prod_{j=1}^{n} \Gamma\left(q_{j}\right)\right) \sum_{\alpha \geqslant 0} \frac{\alpha !}{\left(\Pi_{j=1}^{n} \Gamma\left(q_{j}+\alpha_{j}\right)\right)}\left|b_{\alpha}\right|^{2} .
$$

Again, this is a functional Hilbert space of holomorphic functions in $P$ whose reproducing kernel is

$$
K_{\mathrm{q}}(z, \zeta)=\prod_{j=1}^{n}\left(1-z_{j} \bar{\zeta}_{j}\right)^{-q_{j}} ; \quad z, \zeta \in P
$$

As before, when $q \geqslant 1$ (i.e., when $q_{j} \geqslant 1$ for $j=1, \ldots, n$ ) the norm (7.9) of $\mathcal{H}_{q}$ can be realized as

$$
\|g\|_{\mathrm{q}}^{2}=\frac{1}{\pi^{n}}\left(\prod_{j=1}^{n}\left(q_{j}-1\right)\right) \int_{P}|g(z)|^{2}\left(\prod_{j=1}^{n}\left(1-\left|z_{j}\right|^{2}\right)^{q_{j}-2}\right) d \sigma(z), \quad \mathbf{q} \geqslant \mathbf{1},
$$

and

$$
\|g\|_{\mathbf{q}}^{2}=\frac{1}{(2 \pi)^{n}} \int_{S_{1}^{n}}|g(z)|^{2} d s_{1}\left(z_{1}\right) \cdots d s_{1}\left(z_{n}\right), \quad \mathbf{q}=1,
$$

where in the last inegral, $g$ stands for the nontangential distinguished-boundary values of the holomorphic function $g(z)$ in $P$.

Under these circumstances, the polydisk version of Theorem 1 is

TheOREM 10. Let $f \in \mathscr{D}_{\mathbf{q}}, \mathbf{q}>0$. Then $\exp f \in \mathcal{H}_{\mathbf{q}}$ and

$$
\|\exp f\|_{q}^{2} \leqslant \exp \|f\|_{\mathscr{D}_{q}}^{2} .
$$

Equality holds if and only if $f$ is of the form

$$
f(z)=-\sum_{j=1}^{n} q_{j} \log \left(1-z_{j} \bar{\zeta}_{j}\right), \quad z \in P,
$$

for some $\zeta \in P$.

\section{BiBLIOGRAPHY}

1. V. Bargmann, On a Hilbert space of analytic functions and an associated integral transform. I, Comm. Pure Appl. Math. 14 (1961), 187-214.

2. J. Burbea, A Dirichlet norm inequality and some inequalities for reproducing kernel spaces, Proc. Amer. Math. Soc. 83 (1981), 279-285.

3. , Inequalities for reproducing kernel spaces, Illinois J. Math. 27 (1983).

4. W. F. Donoghue, Jr., Reproducing kernel spaces and analytic continuation, Rocky Mountain J. Math. 10 (1980), 85-97.

5. D. J. Newman and H. S. Shaprio, Certain Hilbert spaces of entire functions, Bull. Amer. Math. Soc. 72 (1966), 971-977.

6. W. Rudin, Function theory in the unit ball of $\mathbf{C}^{n}$, Springer-Verlag, New York, 1980. 
7. S. Saitoh, Some inequalities for analytic functions with a finite Dirichlet integral on the unit disk, Math. Ann. 246 (1979), 69-77.

8. Some inequalities for entire functions, Proc. Amer. Math. Soc. 80 (1980), 254-258.

9. A. Selberg, Automorphic functions and integral operators, Seminars on Analytic Functions, Vol. II, Institute for Advanced Study, Princeton, N. J., 1957, 152-161.

10. I. N. Sneddon, Special functions of mathematical physics and chemistry, Oliver and Boyd, Edinburgh, 1961.

Department of Mathematics and Statistics, University of Pittsburgh, Pittsburgh, PennsylVANIA 15260 